Supporting Information

\title{
Biologically Inspired Polydopamine Capped Gold Nanorods for Drug Delivery and Light-Mediated Cancer Therapy
}

Shaowei Wang ${ }^{l}$, Xinyuan Zhao ${ }^{2}$, Shaochuan Wang ${ }^{l}$, Jun Qian ${ }^{l, *}$, Sailing He ${ }^{l, *}$

${ }^{1}$ State Key Laboratory of Modern Optical Instrumentations, Centre for Optical and Electromagnetic Research, and ${ }^{2}$ Bioelectromagnetics Laboratory, School of Medicine, Zhejiang University, Hangzhou, Zhejiang 310058, China

*Corresponding authors e-mail addresses: sailing@kth.se \& qianjun@zju.edu.cn. 


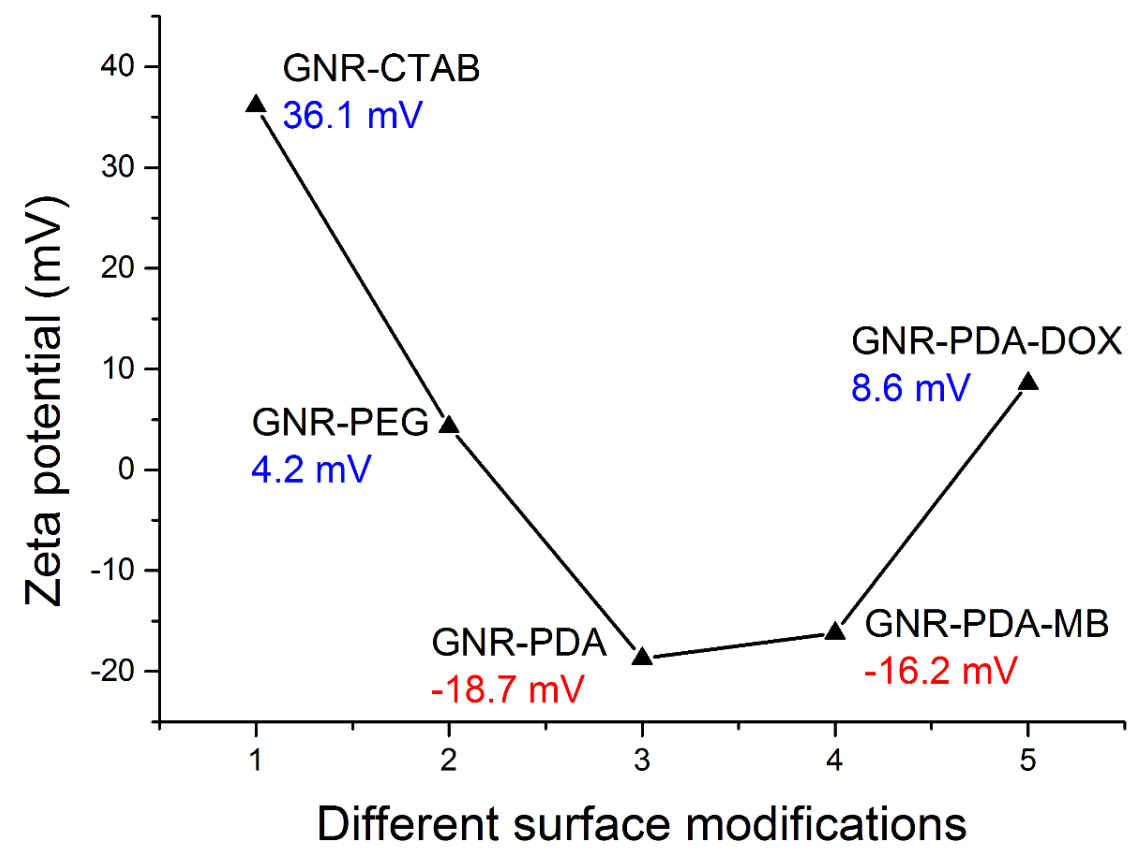

Figure S1. Zeta potentials of GNRs with different surface modifications.

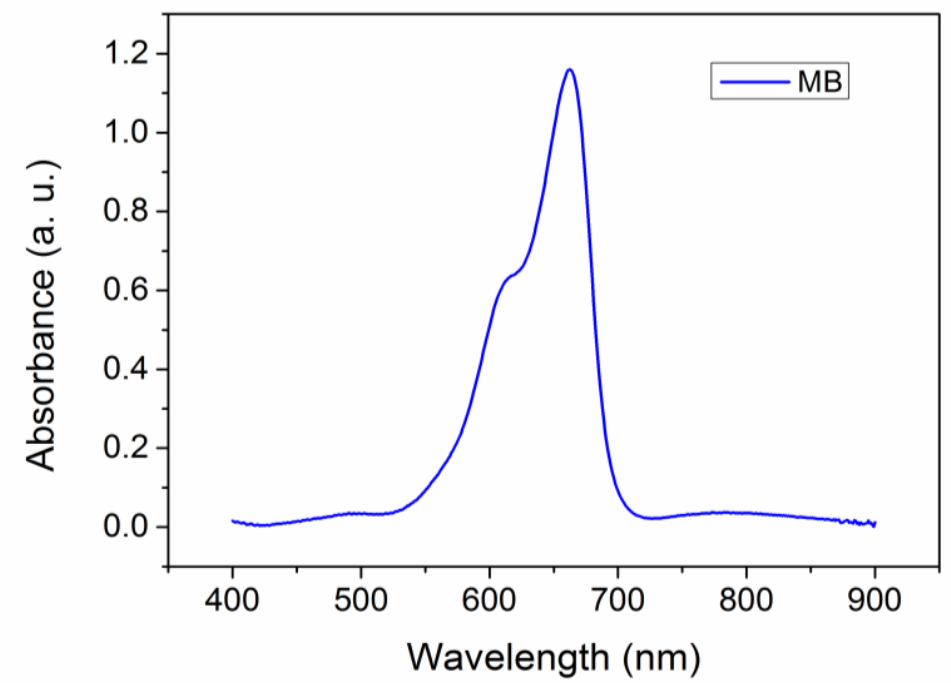

Figure S2. Absorption spectrum of methylene blue (MB) in aqueous solution. 


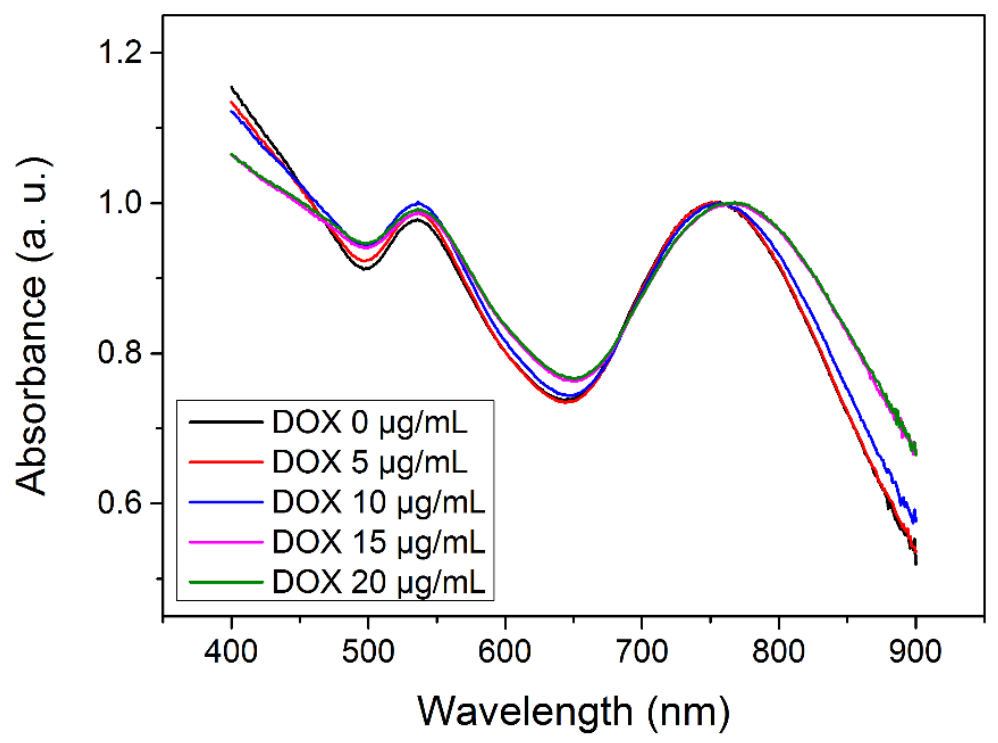

Figure S3. Absorption spectra of GNR-PDA-DOX nanocomposites with various adorbed concentrations of DOX. The corresponding loading efficiencies are $0 \%, 13.1 \%, 26.3 \%, 39.5 \%$, and $52.6 \%(\mathrm{w} / \mathrm{w})$.

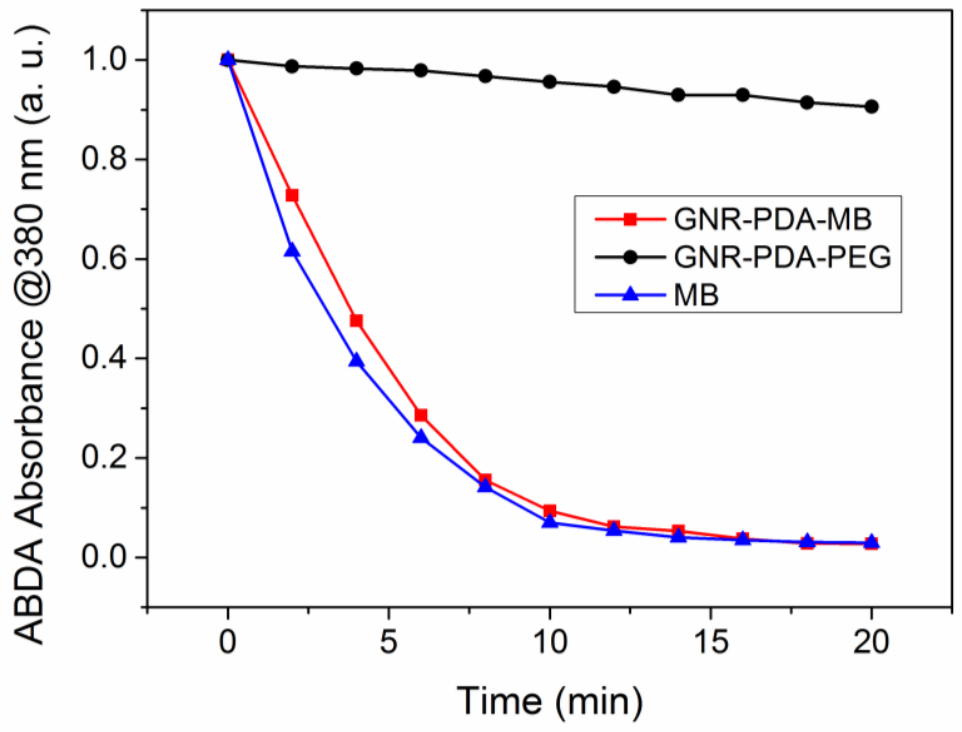

Figure S4. Normalized absorption intensity variation of ABDA at $380 \mathrm{~nm}$ after photo-degradation by ROS, which was generated by GNR-PDA-MB, GNR-PDA-PEG and free MB upon the irradiation of $671 \mathrm{~nm}$-laser $\left(30 \mathrm{~mW} / \mathrm{cm}^{2}\right)$. 


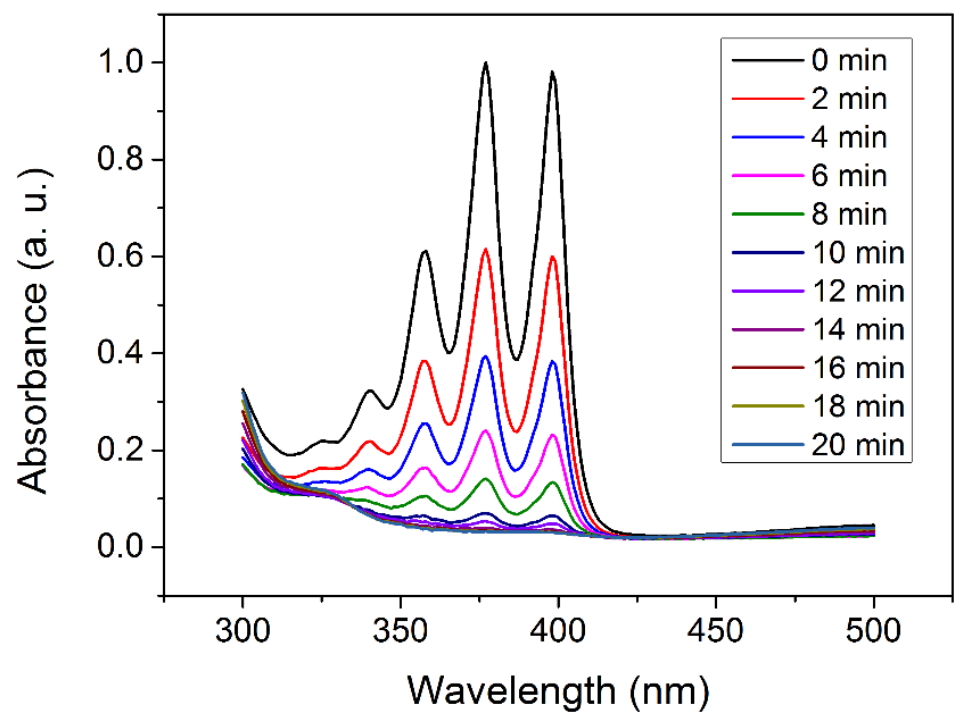

Figure S5. Absorption spectra of ABDA after photo-degradation by ROS, which was generated from free MB $(50 \mu \mathrm{M})$ upon NIR laser irradiation $\left(671 \mathrm{~nm}, 30 \mathrm{~mW} / \mathrm{cm}^{2}\right)$.

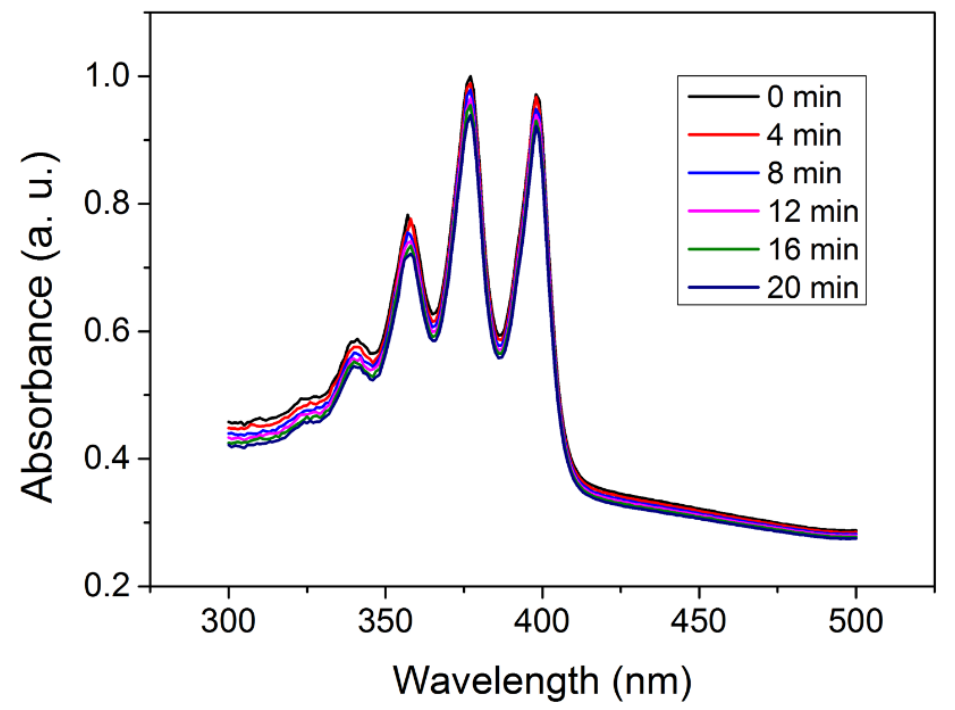

Figure S6. Absorption spectra of ABDA after photo-degradation by ROS, which was generated from GNR-PDA nanocomplex upon NIR laser irradiation $\left(671 \mathrm{~nm}, 30 \mathrm{~mW} / \mathrm{cm}^{2}\right)$. 

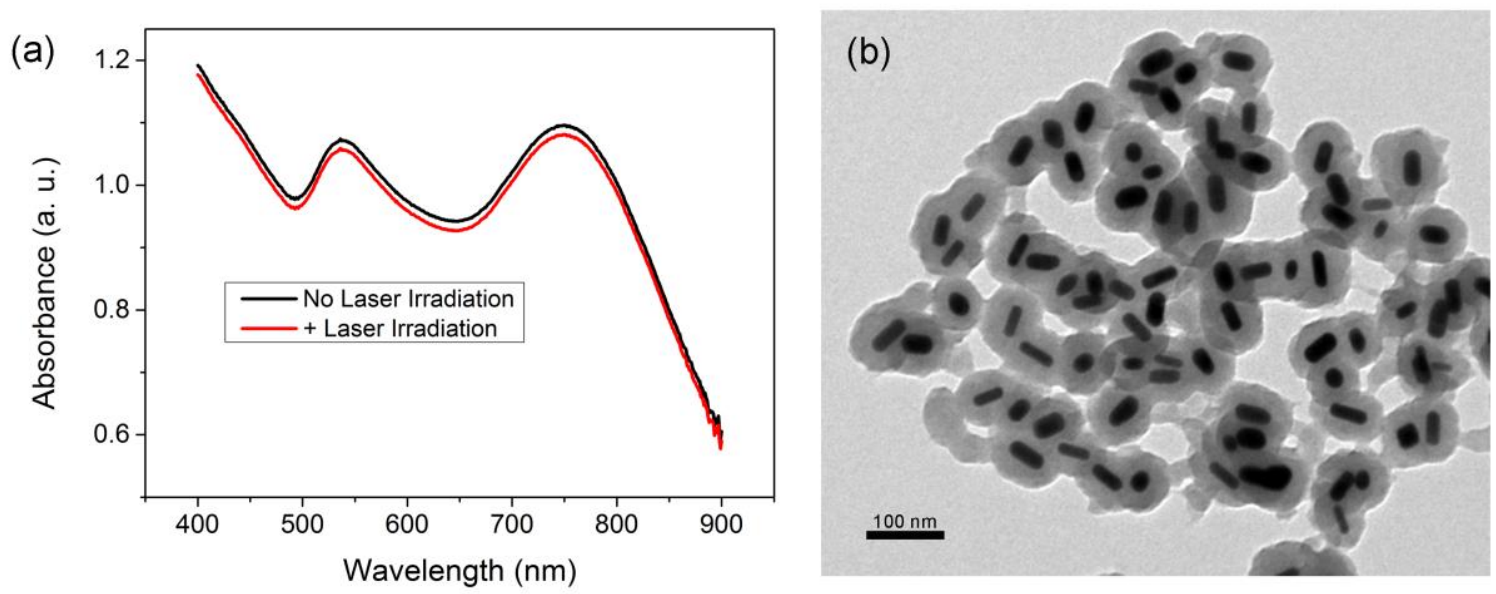

Figure S7. (a) Absorption spectra of aqueous dispersion of GNR-PDA-PEG before and after laser irradiation $\left(808 \mathrm{~nm}, 2 \mathrm{~W} / \mathrm{cm}^{2}\right)$. (b) A TEM image of GNR-PDA-PEG after $1 \mathrm{~h}$ laser irradiation.

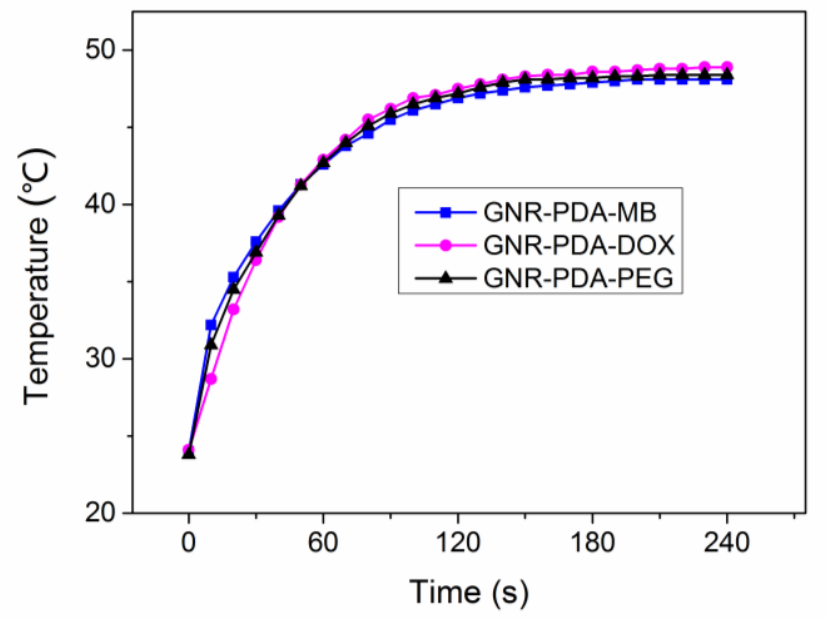

Figure S8. Temperature variation curves of aqueous dispersions of GNR-PDA-MB, GNR-PDA-DOX and GNR-PDA-PEG with the same concentration of $0.1 \mathrm{nM}$, upon the irradiation of NIR laser $\left(808 \mathrm{~nm}, 2 \mathrm{~W} / \mathrm{cm}^{2}\right)$. 


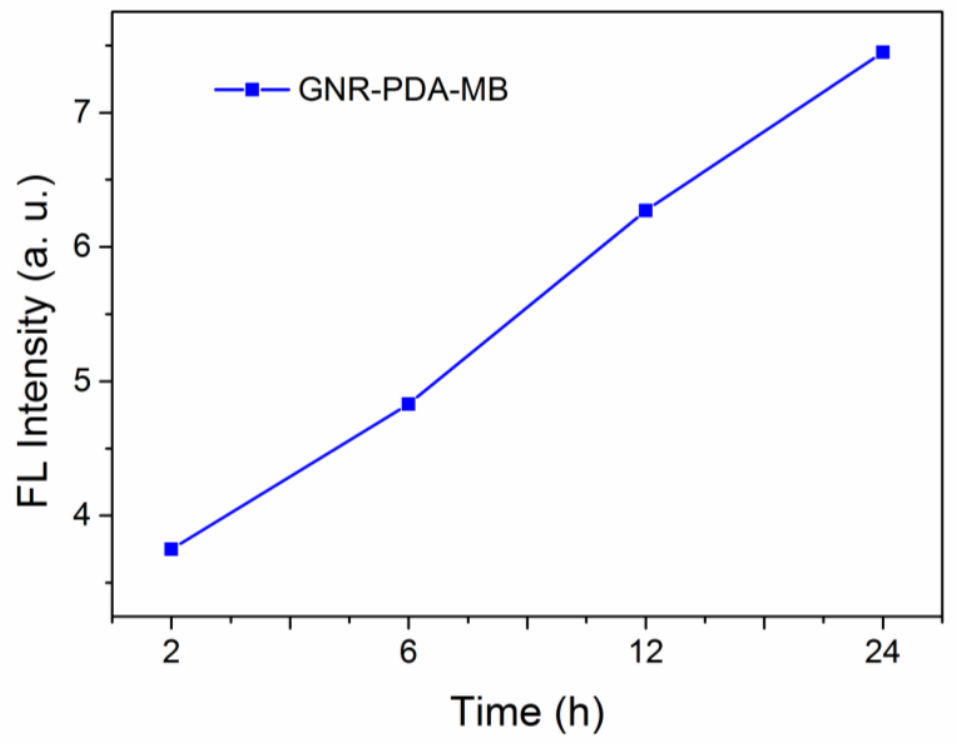

Figure S9. Evaluation of cellular uptake of GNR-PDA-MB nanocomposites, which was determined by flow cytometry. Excitation wavelength, $633 \mathrm{~nm}$.

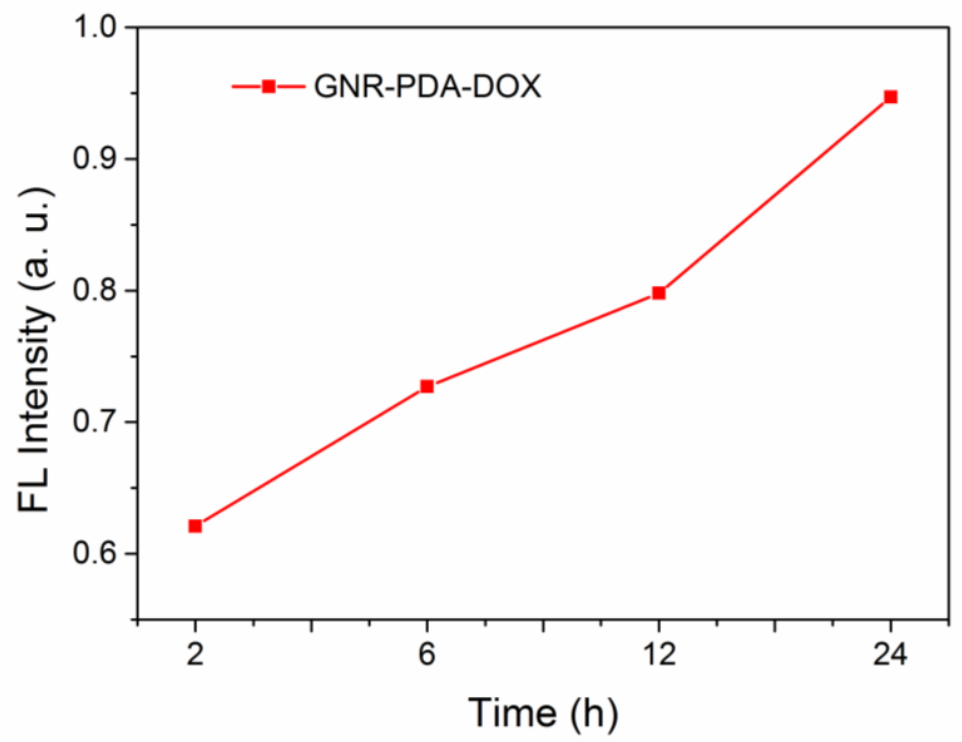

Figure S10. Evaluation of cellular uptake of GNR-PDA-DOX nanocomposites, which was determined by flow cytometry. Excitation wavelength, $488 \mathrm{~nm}$. 

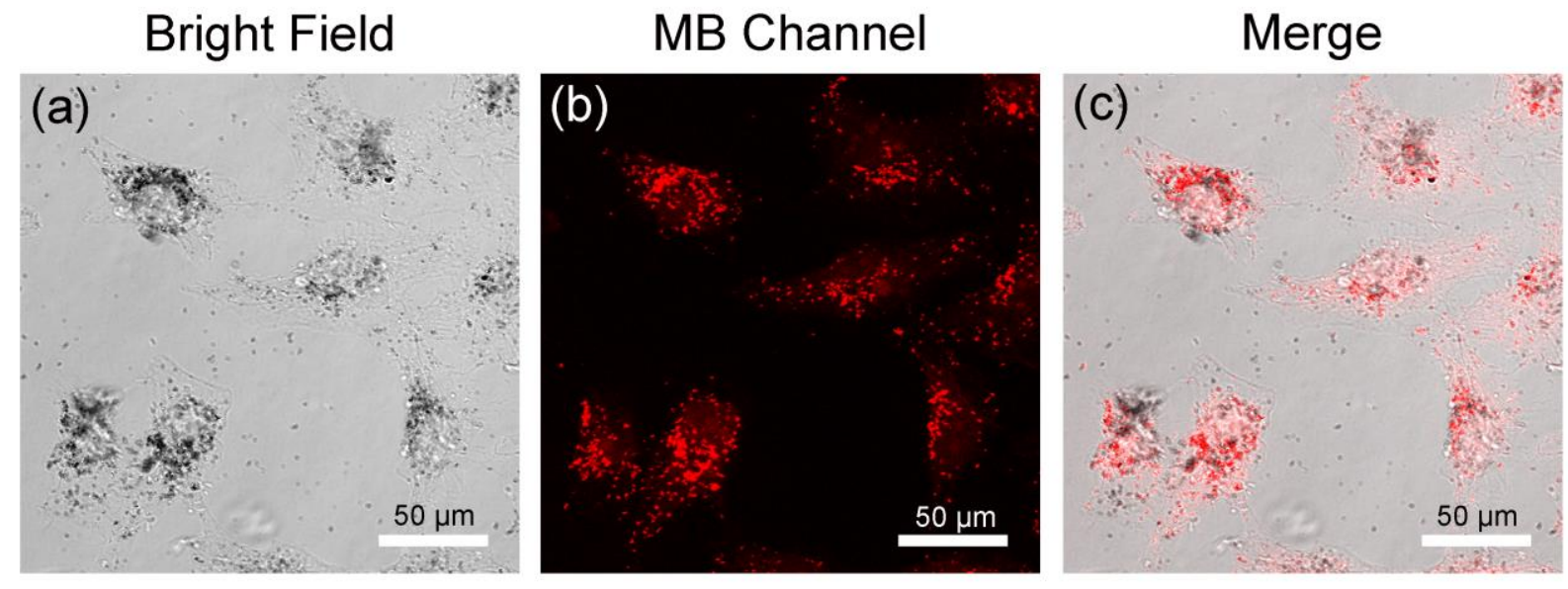

Figure S11. (a) Bright field and (b) fluorescent images of HeLa cells, which were treated with GNR-PDA-MB nanocomposites. (c) the merge image of (a) and (b). Fluorescence (indicated as red) from GNR-PDA-MB was excited by a CW laser at $635 \mathrm{~nm}$ and collected within $655-755 \mathrm{~nm}$ spectral range. 


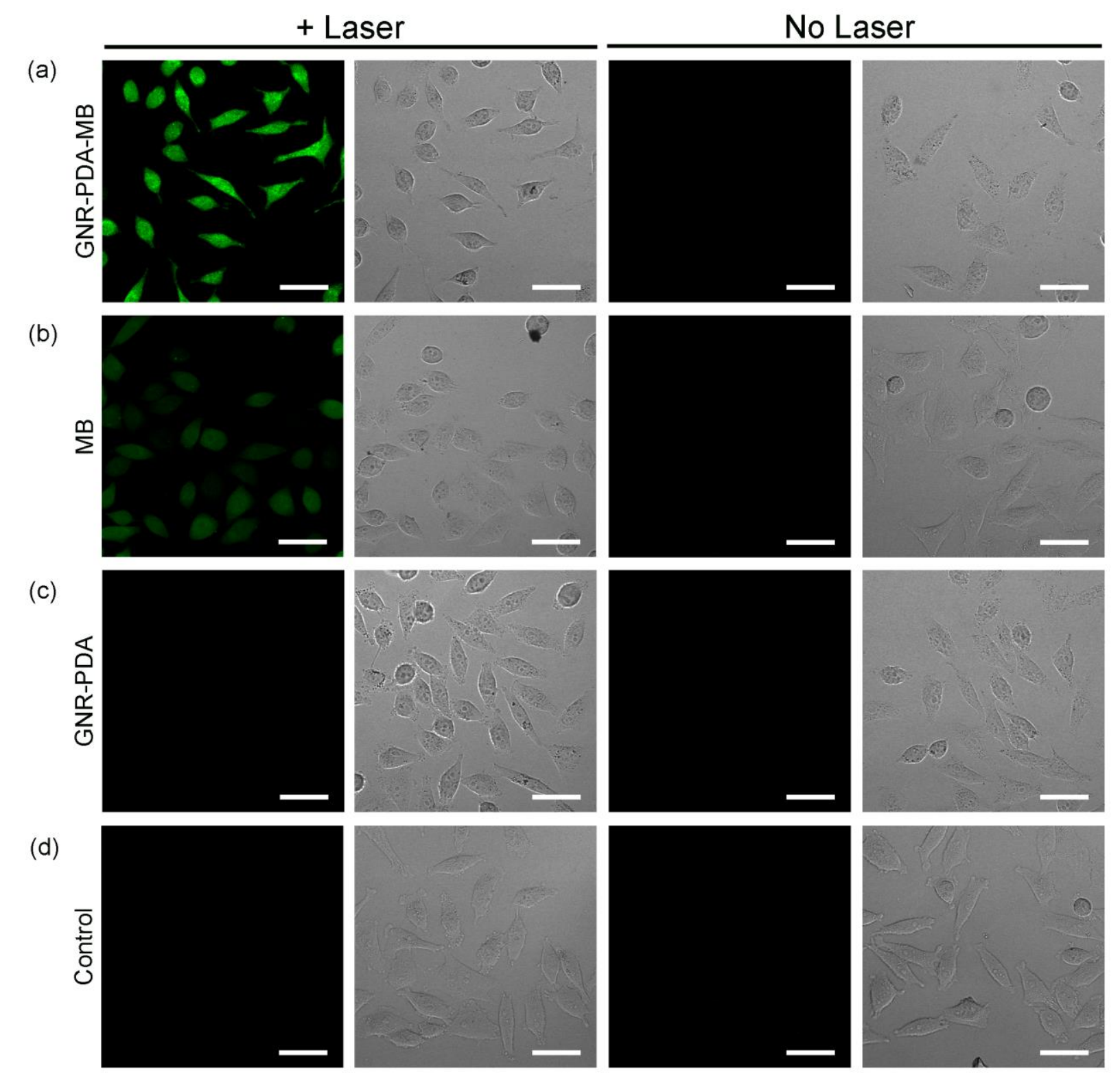

Figure S12. Fluorescent images of HeLa cells, which were incubated with (a) GNR-PDA-MB, (b) free MB, (c) GNR-PDA and (d) PBS, and received laser irradiation or no laser irradiation as indicated. Fluorescence (indicated as green) came from DCF (generated when ROS indicator DCFH-DA reacted with ROS), and the excitation source was a CW laser at $488 \mathrm{~nm}$. Scale bars, $50 \mu \mathrm{m}$. 


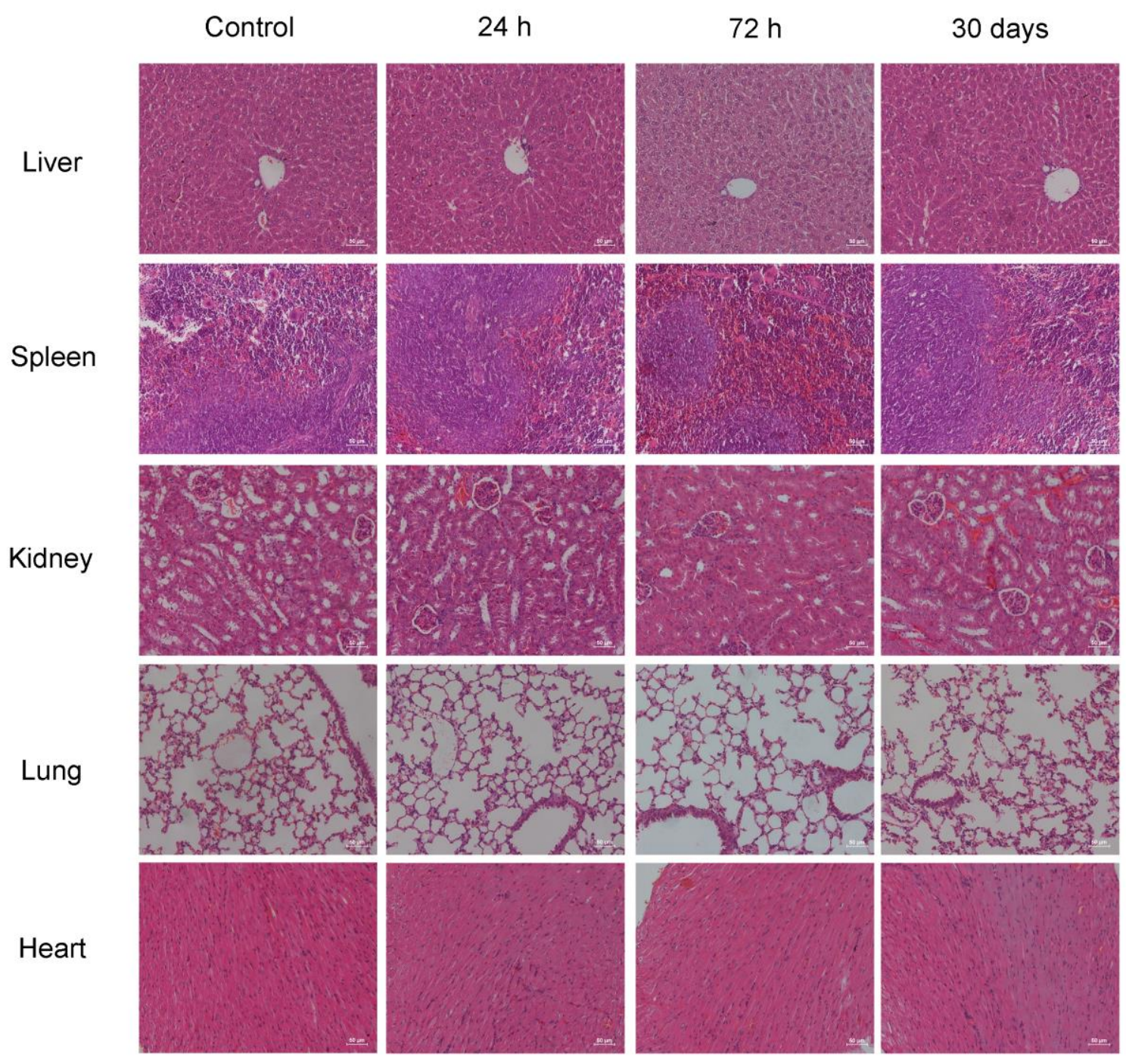

Figure S13. Histological examination of liver, spleen, kidney, lung and heart stained with haematoxylin and eosin. All tissues were collected from mice euthanized at $24 \mathrm{~h}, 72 \mathrm{~h}$ and 30 days after the administration of $200 \mu \mathrm{L}$ of GNR-PDA-MB ( $1 \mathrm{nM}$ in $1 \times$ PBS). The control mice were treated with $200 \mu 11 \times$ PBS. Scale bars: $50 \mu \mathrm{m}$. 


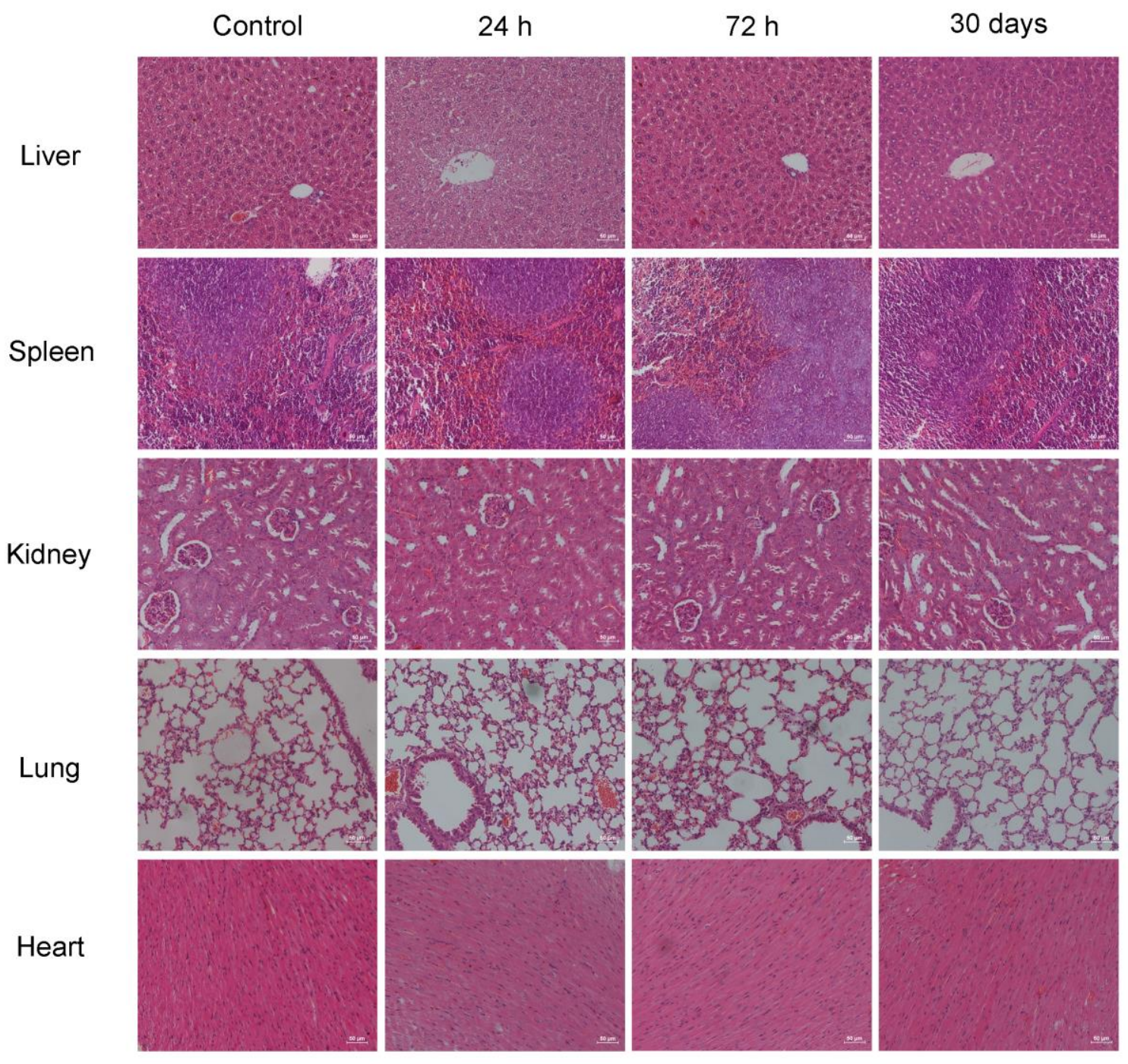

Figure S14. Histological examination of liver, spleen, kidney, lung and heart stained with haematoxylin and eosin. All tissues were collected from mice euthanized at $24 \mathrm{~h}, 72 \mathrm{~h}$ and 30 days after the administration of $200 \mu \mathrm{L}$ of GNR-PDA-DOX $(1 \mathrm{nM}$ in $1 \times$ PBS $)$. The control mice were treated with $200 \mu 11 \times$ PBS. Scale bars: $50 \mu \mathrm{m}$. 